\title{
Some anti-solutions of the Pillai's conjecture and proof of Fermat's Last Theorem.
}

\section{PROF. DR. K. RAJA RAMA GANDHI ${ }^{1}$, REUVEN TINT ${ }^{1}$, MICHAEL TINT ${ }^{2}$}

Resource person in Math for Oxford University Press and Professor at BITS-Vizag ${ }^{1}$

Number Theorist, Israel ${ }^{1}$

Software Engineer, Israel ${ }^{2}$

Email: editor126@gmail.com, reuven.tint@gmail.com, tintmisha@gmail.com

Abstract. Let us show several; including the part already well known variants of find uncountable set solutions of equation

$$
\mathrm{A} x^{m}-B y^{n}=C[\mathbf{1}]
$$

for natural numbers $A, B, C, x, y, m, n$ of specified values $A, B, C$,in contrast to the Pillai's conjecture, in which it is assumed that the set of solutions of equation [1] is finite, and proof of Fermat's Last Theorem.

\section{Solution}

\section{$\S 1$}

$$
m=n=1
$$

If the numbers $\mathrm{A}$ and $\mathrm{B}$ are coprime $[(A, B)=1]$, then the equation

$$
A x+B y=C
$$

always has results in integers (positive) integer numbers and its solutions will always be all the pairs $\left(x_{t}, y_{t}\right)$, where

$$
\begin{gathered}
x_{t}=C \times A^{\varphi(B)-1}+B \times t, \\
y_{t}=C \times \frac{1-A^{\varphi(B)}}{B}-A \times t,
\end{gathered}
$$

$t$ - any integer,

$\varphi(B)$ - Euler function.

N.N. Vorobiev, "Divisibility" (3) Science Glavfizmatgiz, Moscow, 1974, p. 47.

\section{$\S 2$}

2.1.

$$
m=2 ; n=2 ; A=1 ; C=1 \text {. }
$$


The equation

$$
x^{2}-B y^{2}=1
$$

where $" \mathrm{~B} "=D$ is not exact square, has an infinite set of solutions and all of its solutions in the smallest natural numbers are the formulas:

$$
\begin{gathered}
x_{k+1}=x_{1} x_{k}+D y_{1} y_{k} \\
y_{k+1}=y_{1} x_{k}+x_{1} y_{k} \quad(k=1,2,3,4, \ldots) .
\end{gathered}
$$

For $D=2,3,5,6,7,8,10,11,12,13,29 \ldots$ and etc.

$$
\begin{gathered}
(x, y)=(3,2),(2,1),(9,4),(5,2),(8,3), \\
(3,1),(19,6),(10,3),(7,2),(649,180),(4901,1820) \text { and etc. }
\end{gathered}
$$

2.1.1. Then it is possible another variant

$$
A x^{2}-y^{2}=1 ;\left(x_{0} \sqrt{A}+y_{0}\right)^{k}=x \sqrt{A}+y
$$

where $k$ is odd number.

$$
\begin{gathered}
A=2 ; x_{0}=1 ; y_{0}=1, k=7 \\
x=13^{2}=169 ; y=239 ; 2 \times 13^{4}-239^{2}=1 .
\end{gathered}
$$

2.2. The complete solution issue see. V.Sierpinski, "The solution of equations in integers" (1) Fizmatgiz, Moscow, 1961, pp. 29-33..

A.O. Gelfond, "Solving equations in integers" (2) "Science", Glavfizmatgiz, Moscow, 1978.

\section{$\S 3$}

3.1.

$$
m=n=2 ; c=1
$$

The equation

$$
A x^{2}-B y^{2}=1
$$

where $A$ and $B$ is not exact square, has an infinite set of solutions are natural (whole) numbers, which can be found from the equation

$$
x \sqrt{A}+y \sqrt{B}=\left(x_{0} \sqrt{A}+y_{0} \sqrt{B}\right)^{k}[2]
$$

where $k$ - is odd number.

3.2. The equations

$$
\begin{gathered}
3 x^{2}-2 y^{2}=1 ; \quad(A-B=1) \\
x \sqrt{3}+y \sqrt{2}=(1 \times \sqrt{3}+1 \times \sqrt{2})^{k},
\end{gathered}
$$


since they have an obvious solution $(1,1)$.

Put " $k$ " values $3,5,7 \ldots$, we obtain $(9,11),(89,109)$ and etc.

3.3. U.S. Davydov, "Exercises in theoretical arithmetic of integers", the Ministry of Education Uchpedgiz BSSR, Minsk, 1963, N266, p.61 (4).

\section{$\S 4$}

\section{Statement}

4.1. "For each an arbitrary set of four natural numbers

$$
x^{m}, y^{n}, p^{k}, q^{t}
$$

where all 8 parameters not related to each other (autonomous), there are at least two equations, such that

$$
\left\{\begin{array}{l}
A x^{m}-B y^{n}=C \\
A p^{k}-B q^{t}=C
\end{array}\right.
$$

where $A, B, C$-are natural numbers (for each equation are the same), the values of which depend only on the values $x^{m}, y^{n}, p^{k}, q^{t "}$.

\section{Proof.}

4.2. If

$$
A \vartheta_{1}-B u_{1}=C[4]
$$

Then

$$
\begin{gathered}
\left\{\begin{array}{l}
\vartheta_{2}=\vartheta_{1}+B \\
u_{2}=u_{1}+A
\end{array}[5]\right. \\
A \vartheta_{2}-B u_{2}=C[6] .
\end{gathered}
$$

Can be verified directly by substituting $[5]$ in $[6]$.

4.3. From $[5]$

$$
\left\{\begin{array}{l}
B=\vartheta_{2}-\vartheta_{1} \\
A=u_{2}-u_{1}
\end{array}\right.
$$

Substituting in [4] and [6], we obtain

$$
\left\{\begin{array}{l}
\left(u_{2}-u_{1}\right) \vartheta_{1}-\left(\vartheta_{2}-\vartheta_{1}\right) u_{1}=C \\
\left(u_{2}-u_{1}\right) \vartheta_{2}-\left(\vartheta_{2}-\vartheta_{1}\right) u_{2}=C
\end{array}[\text { [8] }\right.
$$

and

$$
C=u_{2} \vartheta_{1}-\vartheta_{2} u_{1}
$$


4.4. Let

$$
u_{2}=5^{3} ; u_{1}=2^{3} ; \vartheta_{2}=3^{4} ; \vartheta_{1}=4^{3}
$$

Therefore,

$$
\left\{\begin{array}{l}
\left(5^{3}-2^{3}\right) 4^{3}-\left(3^{4}-4^{3}\right) 2^{3}=7352 \\
\left(5^{3}-2^{3}\right) 3^{4}-\left(3^{4}-4^{3}\right) 5^{3}=7352
\end{array}\right.
$$

and

$$
\begin{aligned}
& 117 \times 3^{4}-17 \times 5^{3}=7352 \\
& 117 \times 4^{3}-17 \times 2^{3}=7352
\end{aligned}
$$

This completes the proof of statement.

P.S. If $\left(\vartheta_{1}, \vartheta_{2}, u_{1}, u_{2}\right)$ - arbitrary natural numbers in even powers such that $\left(\vartheta_{2}-\vartheta_{1}\right) ;\left(u_{2}-\right.$ $\left.u_{1}\right)$ - are inexact squares, then using the method $\$ \mathbf{5}$, can be get infinite set of solutions of equations [8] we say that rational numbers for each fixed triple $A, B, C$.

\section{$\S 5$}

5.1. We obtain identities:

$$
A(A+3 B)^{2}-B(3 A+B)^{2} \equiv(A-B)^{3}[\mathbf{1 0}]
$$

and

$$
\frac{A}{(A-B)^{3}}(A+3 B)^{2}-\frac{B}{(A-B)^{3}}(3 A+B)^{2} \equiv 1[\mathbf{1 1}]
$$

5.2. Since [11] we have

$$
\begin{gathered}
x \sqrt{\frac{A}{(A-B)^{3}}}+y \sqrt{\frac{B}{(A-B)^{3}}}= \\
=\left(x_{0} \sqrt{\frac{A}{(A-B)^{3}}}+y_{0} \sqrt{\frac{B}{(A-B)^{3}}}\right)^{k}= \\
=\left[(A+3 B) \sqrt{\frac{A}{(A-B)^{3}}}+(3 A+B) \sqrt{\frac{B}{(A-B)^{3}}}\right]^{k}[\mathbf{1 2}],
\end{gathered}
$$

where " $k "$-is arbitrary odd natural number. 
5.3. For every specific value $A$ and $B$, where $A, B$-are inexact squares of arbitrary natural numbers, such as,

1) $A$ and , $B$ such that both are odd

$$
A-B=2 \text {. }
$$

Similarly, the equation [12] provides infinite set of solutions" $x$ " and " $y$ " of natural numbers for each pair $A$ and $B$ for constant

$$
A, B, C=(A-B)^{3} \text {. }
$$

2) In the case, for example, where $A$ and $B$ are numbers with different parity (inexact squares), the equation [12] provides infinite set of solutions " $x$ " and " $y$ " of rational numbers for each pair $A$ and $B$ for constant

$$
A, B, C=(A-B)^{3} \text {. }
$$

\section{Examples.}

If $A=5 ; B=3 ; k=3$;from [13]

$$
\begin{gathered}
x_{0}=A+3 B=5+3 \times 3=14 ; \\
y_{0}=3 A+B=3 \times 5+3=18 \\
5 \times 14^{2}-3 \times 18^{2}=8
\end{gathered}
$$

5.4. Follows from $[15]$

$$
\begin{gathered}
x \sqrt{\frac{5}{8}}+y \sqrt{\frac{3}{8}}=\left(14 \sqrt{\frac{5}{8}}+18 \sqrt{\frac{3}{8}}\right)^{3}= \\
14^{3} \times \frac{5}{8} \times \sqrt{\frac{5}{8}}+3 \times 14^{2} \times \frac{5}{8} \times 18 \times \sqrt{\frac{3}{8}}+ \\
+3 \times 14 \sqrt{\frac{5}{8}} \times 18^{2} \times \frac{3}{8}+18^{3} \times \frac{3}{8} \sqrt{\frac{3}{8}} \\
x=14^{3} \times \frac{5}{8}+3 \times 14 \times 18^{2} \times \frac{3}{8}= \\
=5 \times 7^{3}+3^{6} \times 7=6818 \\
y=3 \times 14^{2} \times \frac{5}{8} \times 18+18^{3} \frac{3}{8}=
\end{gathered}
$$




$$
=3^{3} \times 5 \times 7^{2}+3^{7}=8802
$$

and

$$
\begin{aligned}
& 5 \times 6818^{2}-3 \times 8802^{2}=(5-3)^{3}=2^{3}= \\
& =232425620-232425612=8 \text { and etc. }
\end{aligned}
$$

5.5. Thus,

$$
A x^{2}-B y^{2}=C ; 5 x^{2}-3 y^{2}=8[13]
$$

where $x, y$ - are infinite numbers of the equation [13] of natural numbers for constant $A=$ 5; $B=3 ; C=8$ for the case where $C \neq 1$.

§6

6.1. We obviously have the identity:

$$
\left(y^{t}+k\right)^{z-q}\left(y^{t}+k\right)^{q}-y^{t} \times \frac{\left(y^{t}+k\right)^{z}-k^{z}}{\left(y^{t}+k\right)-k} \equiv k^{z}[\mathbf{1 4}]
$$

6.2. If

$Z$-is inexact square arbitrary odd natural number;

$q-$ is even natural number;

$t$ - is even natural number;

$(z-q)-$ is inexact square;

" $k "$ - arbitrary natural number,

then using the method of the previous section, we can get infinite set of solutions, we shall say that, of rational numbers in the equation $[\mathbf{1 4}]$ for constant $A, B, C$.

\section{$\S 7$}

The proof of the Fermat's Last Theorem (another version)

7.1. Let us prove that in the equation

$$
x^{n}+y^{n}=z^{n}[\mathbf{1 5}]
$$

$x_{0}, y_{0}, z_{0}$-are solution of [15] in natural numbers for $n>2$.

7.2. Then, follows from [15]

$$
\begin{gathered}
z^{n}-x^{n}=y^{n} \\
z^{m} z^{n-m}-x^{z} x^{n-r}=y^{n}
\end{gathered}
$$




$$
\frac{z^{m}}{y^{n}} z^{n-m}-\frac{x^{r}}{y^{n}} x^{n-r}=1[\mathbf{1 6}]
$$

where $n>2$ - is arbitrary odd natural number;

$m<n, r<n-$ are inexact squares arbitrary odd natural numbers.

$n-m=2 p, n-r=2 q$

$$
\frac{z_{0}^{m}}{y_{0}^{n}}\left(z_{0}^{p}\right)^{2}-\frac{x_{0}^{r}}{y_{0}^{n}}\left(x_{0}^{q}\right)^{2}=1[17] .
$$

7.3. Using the method of $\$ \mathbf{5}$,we have

$$
z \times \sqrt{\frac{z_{0}^{m}}{y_{0}^{n}}}-x \times \sqrt{\frac{x_{0}^{r}}{y_{0}^{n}}}=\left(z_{0}^{p} \times \sqrt{\frac{z_{0}^{m}}{y_{0}^{n}}}-x_{0}^{q} \times \sqrt{\frac{x_{0}^{r}}{y_{0}^{n}}}\right)^{k}[\mathbf{1 8}]
$$

where $k$ - is arbitrary odd natural number, and hence infinite set of solutions $z, x, y$ of equations [18], [17], [16], [15] for each odd $n>2$ in natural (rational) numbers; it is clear that using research of G. Faltings (5) the equation [15] has no solutions in the natural (rational) numbers for arbitrary natural $n>2$, because equations [15] has no solution for $n=4$, as we know, has proven by Fermat himself.

7.4. Indeed, if $3^{x}$ equation having at least one solution $(\mathbf{\$} \mathbf{2}, \mathbf{3}, \mathbf{4 , 5}, \mathbf{6})$ such that allow obtain from it type equation [2], [12], [16], [17], [18] then the solutions of are countless.

7.5. To conclude the proof, it remains to note that with not coprime solutions of $[15]$ in (6), 1.2., 2.1.2.2.2.3. and (7), 2.6.9. We obtain a complete solution to the Fermat's Last Theorem.

7.6. Indeed, as in the general case, in (6) and (7), if

$$
x^{n}+y^{n}=z^{n}
$$

then

$$
\begin{gathered}
x^{n}\left(x^{35} y^{84} z^{20}\right)^{n}+y^{n}\left(x^{35} y^{84} z^{20}\right)^{n}= \\
=z^{n}\left(x^{35} y^{84} z^{20}\right)^{n}[\boldsymbol{I}] \\
{\left[\left(x^{9} y^{21} z^{5}\right)^{4}\right]^{n}+\left[\left(x^{7} y^{17} z^{4}\right)^{5}\right]^{n}=} \\
=\left[\left(x^{5} y^{12} z^{3}\right)^{7}\right]^{n}[\boldsymbol{I I}] \\
{[\boldsymbol{I}]=[\boldsymbol{I I}] .} \\
x^{n}\left(x^{315} y^{224} z^{160}\right)^{n}+y^{n}\left(x^{315} y^{224} z^{160}\right)^{n}= \\
z^{n}\left(x^{315} y^{224} z^{160}\right)^{n}[\boldsymbol{I I I I}]
\end{gathered}
$$




$$
\begin{gathered}
{\left[\left(x^{79} y^{56} z^{40}\right)^{4}\right]^{n}+\left[\left(x^{63} y^{45} z^{32}\right)^{5}\right]^{n}=} \\
=\left[\left(x^{45} y^{32} z^{23}\right)^{7}\right]^{n}[\boldsymbol{I V}] \\
{[\boldsymbol{I I I}]=[\boldsymbol{I} \boldsymbol{V}] \text { and etc. }}
\end{gathered}
$$

These different solutions in parentheses for each natural " $n$ " and each arbitrary fixed triple coprime exponents in the square brackets $(4,5,7)$ - are countless.

7.7. From the initial same, but slightly modified considerations that used in 7.6., we obtain follows identity:

$$
\begin{gathered}
{\left[x^{3 p+1} y^{3 q} z^{2(3 t-2)}\right]^{3 t-2}+\left[x^{3 p} y^{3 q+1} z^{2(3 t-2)}\right]^{3 t-2} \equiv} \\
\equiv\left[x^{3 p} y^{3 q} z^{3(2 t-1)}\right]^{3 t-2}[\boldsymbol{V}]
\end{gathered}
$$

if

$$
x^{3 t-2}+y^{3 t-2}=z^{3 t-2}
$$

Here, there are (independent from each other),

$$
\begin{aligned}
p & =1,2,3,4, \ldots \\
q & =1,2,3,4 \ldots \\
t & =1,2,3,4 \ldots \\
3 t-2 & =n=1,4,7,10, \ldots
\end{aligned}
$$

7.8. The identity of type $[\boldsymbol{V}]$ the same arguments may be obtained for an arbitrary natural" $n$ ".

7.9. As a consequence of the preceding paragraphs of the section in (7), 1.2. all members of the sequence

$$
\frac{\sqrt[n-1]{\left(x^{n-1}+y^{n-1}\right)^{n}}}{\sqrt[n]{\left(x^{n}+y^{n}\right)^{n-1}}}=\frac{\sqrt[n-1]{z_{n-1}^{n}}}{\sqrt[n]{z_{n}^{n-1}}}[19]
$$

for $n>2$ are only irrational numbers. Therefore, the proof of insolubility in natural numbers of the equation

$$
x^{n}+y^{n}=z^{n}
$$

for $n>2$ in the (7) becomes a complete and fully correct. 


\section{References:}

[1] В. Серпинский, “О решении уравнений в целых числах” Физматгиз, Москва, 1961.ИНТЕРНЕТ БИБЛИОТЕКА: http://ilib.mccme.ru/djvu/serp-int_eq.htm

[2] А.О. Гельфонд, “Решение уравнений в целых числах”, Наука Главфизматгиз, Москва, 1978.

[3] Н.Н. Воробьёв, “Приизнаки делимости”, Наука, Главфизматгиз, Москва, 1974.

[4] У.С.Давыдов, “Задачи и упражнения по теоретической арифметике целых чисел”, Учпедгиз, Минск, 1963.

[5] Faltings G (1983). "Endlichkeitssätze für abelsche Varietäten über Zahlkörpern". Inventiones Mathematicae 73 (3): 349-366. doi:10.1007/BF01388432.

[6] PROF. DR. K. RAJA RAMA GANDHI, Reuven Tint, "Репродуцированное решение Ферма его "Великой" теоремы. (элементарный аспект)", Bulletin of Mathematical Sciences \& Applications ISSN: 2278-9634 Vol. 2 No. 3 (2013), pp. 05-15.

[7] Reuven Tint, "The Identities of Ordinary which Is Leading to the Extraordinary Consequences (Elementary Aspect)", Asian Journal of Mathematics and Applications - ISSN 2307-7743, 2013 